$\mathrm{DOE} / \mathrm{ER} / 53250--3$,j --3

DE92 012376

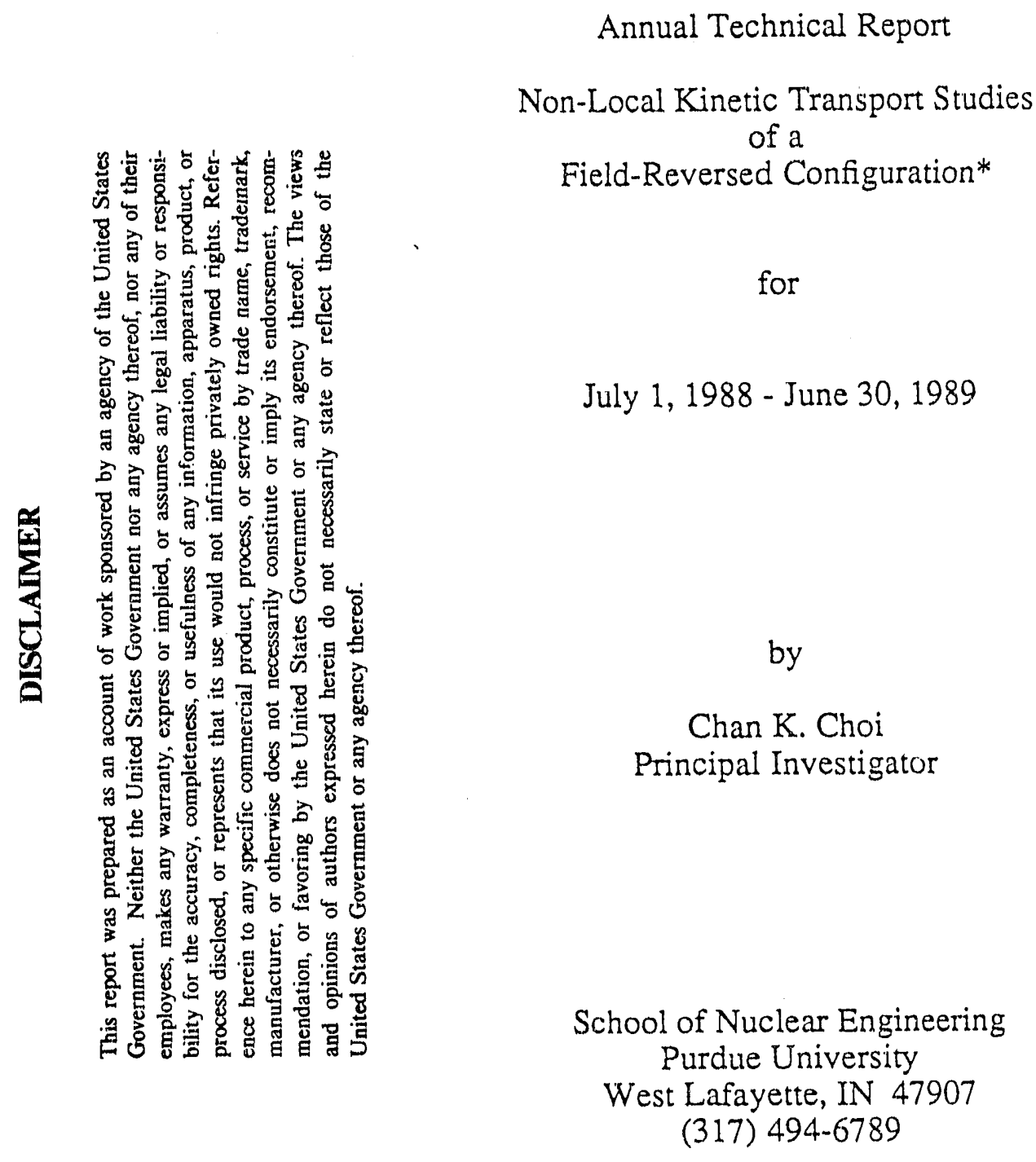

*Work supported by the U.S. Department of Energy under contract DE-FG02-87ER53250.A000. 


\section{THE 2-D EQUILIBRIUM FRC}

One of the positive results that came from the work in this period was the development of a series of codes for solving the 2-D equilibrium FRC problem under various basic physics assumptions. The derivation of the models used to determine an FRC equilibrium was presented in the previous annual report [1], which is to be submitted to Physics of Fluids. The derivation is not repeated in this section; rather, some examples of what has been learned using these codes are presented.

To date, the codes have been useful in four essential areas. First, it was shown that passive mirrors present in the early operation of the LSM device were interacting strongly with the plasma. This caused the inference of some key quantities to be inaccurate. Second, profiles have been found that can exist in the plasma and could cause the present inference of the field null resistivity to be significantly in error. Third, it has become possible to examine rotational effrcts in the FRC equilibrium from the standpoint of modification of both equilibrium and stability properties. Fourth, ir has made possible a realistic study of the MHD stability of the system.

\section{I.1 Early LSM operation}

Early operation of the LSM experiment was conducted with a $10 \mathrm{mTorr} \mathrm{initial} \mathrm{fill} \mathrm{pressure}$ in a flux conserving shell having a $38 \mathrm{~cm}$ inner radius in the midsection and $35 \mathrm{~cm}$ inner radius mirrors at the ends. The flux confinement times as inferred from the experimental data of the plasma radius were about $110 \mu \mathrm{sec}$. This implied an anornaly in the field null resistivity of abuut 8; that is, when using the experimentally inferred current and flux confinement time, the required resistivity was 8 times the Spitzer perpendicular value. It was clear from the plots of 
experimentally measured excluded flux radius that there was a substantial amount of plasma near the mirrors. Previous work by Spencer and Tuszewski [2] had shown that when the end of the FRC is near a mirror there can be a substantial error in the average beta condition. In an effort to determine whether this was occurring in the LSM experiment a search for equilibria that would produce the observed excluded flux radii was made. Figures I.1 and I.2 show the results of such a fit. Figure I.1 shows the experimentally observed excluded flux radii (points) and the excluded flux radii generated with the MHD equilibrium code (solid line). The lack of agreement between the experimental points and the solid curve near the mirror stems primarily from the differences in the way in which mirrors are included in the equilibrium code. In the real experiment the mirrors are made by changing the wall radius. In the present version of the code the effect of mirrors is included by varying the flux at the wall as a function of $z\left(\psi_{w}(z)\right)$. $\psi_{w}$ such that when the fields were solved with no plasma present the magnitude of the magnetic field on axis $B(z)$ was in agreement with calculations made by Rej of the vacuum field in the real system.

Figure I.2 shows plots of several important quantities versus radius in the axial midplane. The quantities are in dimensionless code units with $\psi$ at the wall and the wall radius $r_{w}$ in the midplane normalized to 1 . The contour plots show surfaces of constant flux in the $(r, z)$ plane. In the MHD model, these are also contours of constant pressure because $p=p(\psi)$. The line plots are $\psi$ (the poloidal flux function), "cur" (the toroidal current density), p (the plasma pressure), and "ptot" (the quantity $\left.\left(\mathrm{p}+\mathrm{B}_{\mathrm{z}}^{2} / 8 \pi\right) /\left(\mathrm{B}_{\mathrm{w}}^{2} / 8 \pi\right)\right)$. These plots show that the plasma pressure is very nearly constant over a large region of the plasma interior, and that the current is sharply peaked at the separatrix. The full importance of the quantity $p+B_{z}^{2} /(8 \pi)$ as a diagnostic is discussed in the earlier report [1]. The salient points for the present discussion of "ptot" are that the ringing which can be seen at $r=0.4$ gives us an estimate of the truncation errors present 

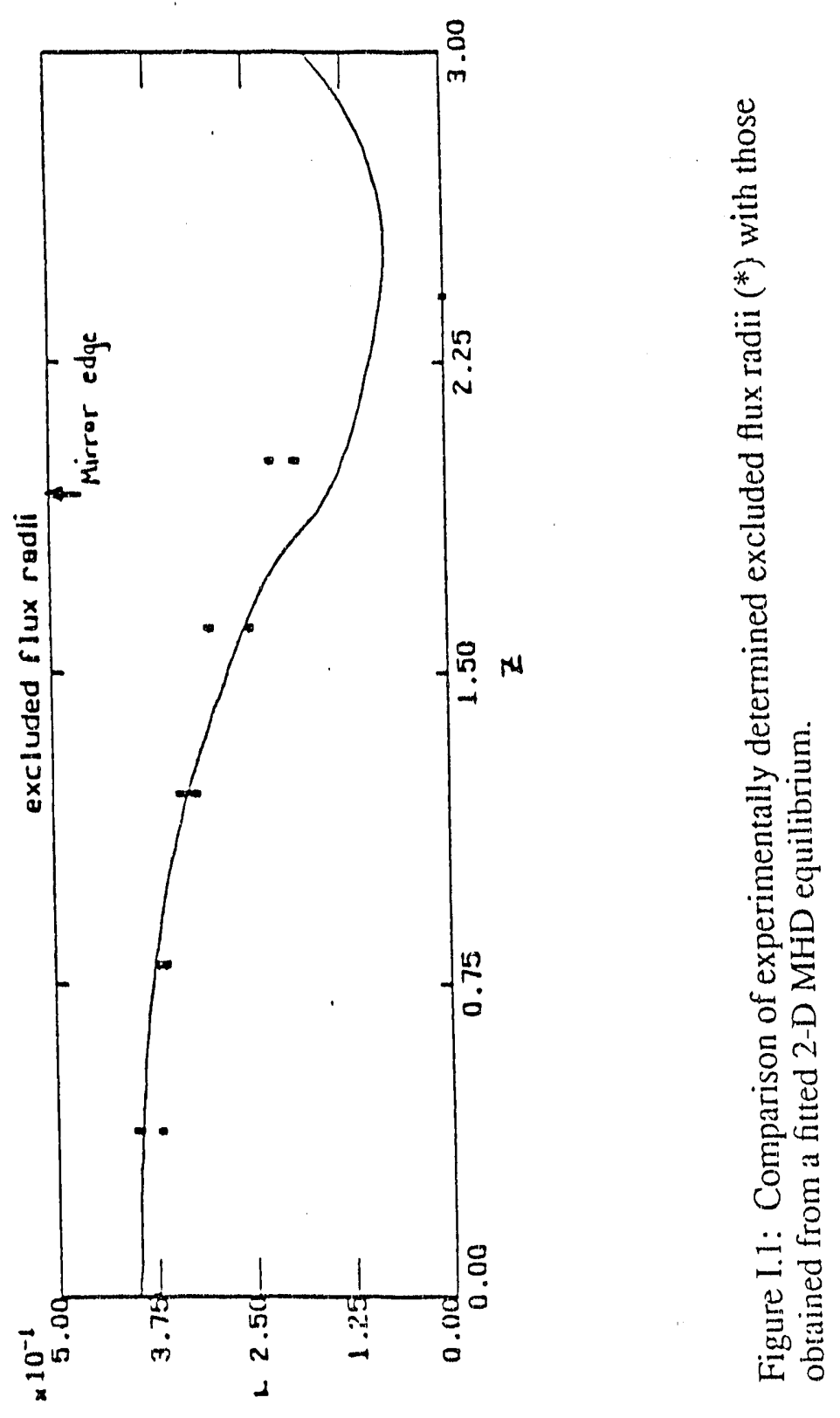

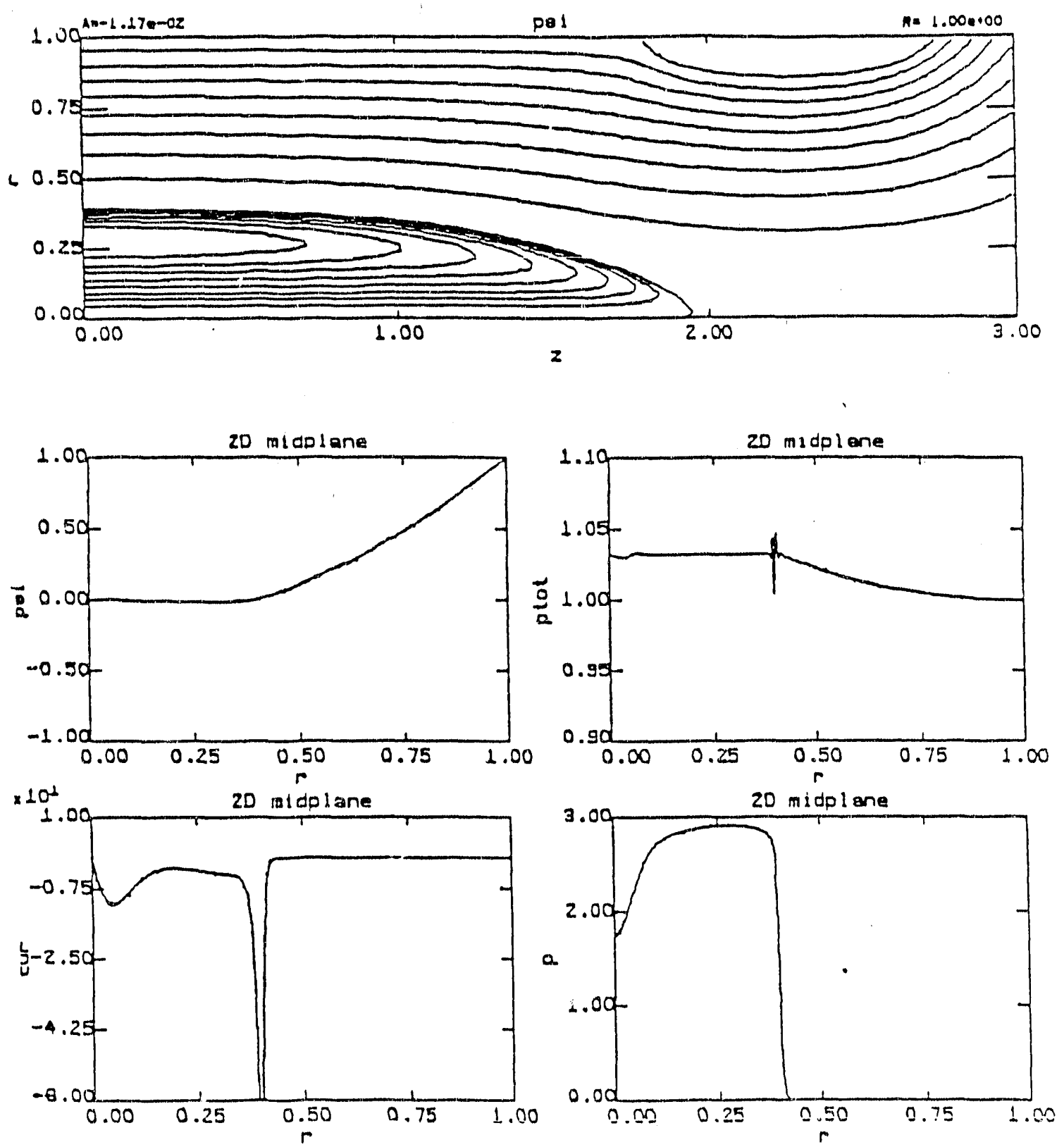

Figure 1.2: Plots showing the nature of the fitted equilibrium. 
int the system, and that the 3 percent drop in pressure from $r=0.4$ to $r=1$ gives an estimate of the correction to $<\beta>$ due to field line curvature. "The level of ringing in the figure "ptot" indicates about a 3 percent local error in $\mathrm{p}+\mathrm{B}_{\mathrm{z}}^{2} /(8 \pi)$. Careful investigation has shown that this error occurs entirely in the magnetic field. The reason for this is that the solution for $\psi$ converges much faster than the solution for $B=\frac{1}{r} \frac{\partial \psi}{\partial r}$ as the grid spacing in the computational model is reduced. This is related to Gibb's phenomenon. It is common in a computational problem to find this behavior. In terms of the physics of the problem, it means the grid is not fine enough to resolve the details of the sheath. These details do not turn out to play a large role in the solution. Experience shows that the solution changes very little once the ringing is below 5 percent peak. ${ }^{1}$

Tuszewski and Spencer [3] give a simple formula for estimating the correction due to field line curvature,

$$
<\beta>_{\mathrm{m}}=1-\mathrm{x}_{\mathrm{s}}^{2} / 2-(\delta / 2)\left(1-\mathrm{x}_{\mathrm{s}}^{2}\right) .
$$

Here $\delta$ is ihe drop in total pressure $\left(p+B_{z}^{2} /(8 \pi)\right)$ from the null to the wall, and

$$
<\beta>_{\mathrm{m}} \equiv \int \mathrm{prdr} / \int \mathrm{p}_{\text {null }} \mathrm{rdr}
$$

Note that the definition of $\langle\beta\rangle_{m}$ is subtly different from the definition of $\langle\beta\rangle$. The quantity $\langle\beta\rangle_{\mathrm{m}}$ is introduced because when curvature is important the trapped flux should be determined using $\langle\beta\rangle_{\mathrm{m}}$ instead of $\langle\beta\rangle$. When curvature is unimportant the two quantities are equal. The approximate effect of field line curvature in the present problem is to reduce $\langle\beta\rangle_{\text {m }}$ by 1.3 percent. This may seem small but for this equilibrium $\langle\beta\rangle_{m}=0.97$ so the important quantity, $\left(1-\langle\beta\rangle_{m}\right)$ that is used to determine the trapped flux is of the same order as this correction.

1. In particular, in every case examined, the trapped flux changes by less than 1 percent once the ringing is below this level. Typically the trapped flux is very sensitive to errors in the algebraic solution of the discretized system. Thus it is a reasonable measure of how well the solution has converged. 
Clearly field line curvature can be important even for long equilibria when the plasma interacts with a mirror.

The original purpose of doing the data fits was to test the accuracy of the experimental inference of the quantity $s=\int_{r_{\text {null }}}^{r_{\text {nop }}}\left(r / r_{s} \rho_{i}\right) d r$ where $\rho_{i}$ is the gyroradius of the ions. The conclusion is that the values of s predicted using the usual diffuse profile model were high by about 40 percent.

This fit does not represent in any way a best fit to the experimental data. No algorithm for fitting a 2-D equilibrium to a given set of experimental data is known. This fit was produced by trial and error, determining input parameters to the 2-D code by hand in order to obtain a better fit. At the time that these equilibria were computed the total particle energy inside the separatrix was used as the constraint for turning the problem of finding a 2-D equilibrium into a nonlinear eigenvalue problem, thereby removing difficulties associated with the fact that most interesting FRC equilibria in an infinite cylinder occur very near a bifurcation point. In retrospect, this was not actually necessary. The presence of the magnetic mirror also removes the bifurcation. The use of the enclosed energy as a constraint led to the observation that the enclosed energy was a crucial parameter in obtaining good fits. In particular, to get the FRC to lean as heavily on the mirror as was observed in the experiment it was necessary to increase the enclosed energy substantially over that typical of an FRC of similar length and profile but not under the influence of the mirrors. ${ }^{2}$ Essentially what happens is that given a profile $p(\psi) / \mathrm{p}_{\text {null }}(\psi)$ as one increases the energy in the plasma the FRC first lengthens axially, holding the midplane cross-section constant. When the end of the FRC gets close to the mirror, the mirror prevents the FRC from

2. A profile is specified by giving the shape function $S$ where $S=p(\psi) / p_{\text {null }}$ with $p_{\text {null }}$ being the pressure at the field null. 
getting longer. Adding energy to the plasma then causes it to get fatter. This qualitative understanding is important. The usual method of deducing the trapped flux from the radius using the average beta condition assumes that the $\mathrm{FRC}$ is free to lengthen or shorten. It is this freedom that allows one to relate the trapped flux to the radius [4]. When the length of the FRC is fixed by mirrors, the radius becomes very sensitive to the energy confined in the plasma. The radius then no longer provides a useful estimate of the flux confinement time. It was known that energy was being lost across the separatrix; this suggested that one should expect the FRC to lean less heavily on the mirrors as time progressed. This was counter to the common opinion at the time. The inferred flux and energy loss rates suggested that the FRC should lean more heavily on the mirror as time increased. The inferred value for $\tau_{\phi}$ was less than the inferred value for $\tau_{E}$. As a result, the predictions of a zero- $D$ code for an FRC without mirrors were that the FRC should get longer. The inferred flux and energy loss rates suggested that the FRC should lean more heavily on the mirror as time increased. This discrepancy led one to do fits to a single good shot at two different times. The results are summarized in Table I.1.

Table I.1: Results of fitting 2-D equilibria to LSM shot 6665.

\begin{tabular}{cll} 
& $30.5 \mu \mathrm{sec}$ & $90.5 \mu \mathrm{sec}$ \\
\hline $\mathrm{x}_{\mathrm{s}}$ & 0.40 & 0.35 \\
$\langle\beta>$ & 0.991 & 1.000 \\
$\left(\mathrm{~B}_{\mathrm{w}} / \mathrm{B}_{\mathrm{F}}\right)^{2}$ & 0.974 & 0.963 \\
$\phi_{\mathrm{H}}$ & 0.0269 & 0.0173 \\
$\phi_{\mathrm{t}}$ & 0.0196 & 0.0122 \\
$\phi_{\mathrm{t}}^{*}$ & 0.0117 & 0.0087
\end{tabular}

The columns refer to the time at which the data were fit. These times are 30.5 and 90.5 microseconds after the reversal of the current in the theta pinch coil. The ratio $\left(B_{w} / B_{F}\right)^{-1}$ is defined to be $\sqrt{8 \pi p_{\text {null }}} / \mathrm{B}_{\mathrm{w}}$. This quantity is used because, at the time that this work was performed, the paper by Tuszewski and Spencer dealing with curvature effects had not yet been 
published. The method for making the correction using $B_{F} \equiv \sqrt{8 \pi p_{n u l l}}$ was developed without knowledge of their work. The methods are essentially the same $\left.\left(\left(B_{w} / B_{F}\right)^{2}<\beta\right\rangle=\langle\beta\rangle_{m}\right)$. The description of the cletermination of $\phi$ follows.

Profile effects are always present in the 2-D calculations. Since the calculations do not involve transport, there is no way to select the proper profile. The usual experimental reduction of data assumes a model profile. It is necessary to assume the same profile when comparing with the experimental inferences. The plot of $\mathrm{p}+\mathrm{B}_{\mathrm{z}}^{2} /(8 \pi)$ (ptot) in Figure I.2 shows that field line curvature is negligible inside the FRC. Experience with the $2 \mathrm{D}$ codes also indicates that the open field line plasma has a negligible impact on determining the external magnetic fields; that is, the magnetic field outside the separatrix. Consideration of the derivation of the average beta condition shows that if the field line curvature inside the FRC and the open field line pressure are negligible, $\langle\beta\rangle$ is a function only of the shape of the separatrix and the flux conserver.

Similarly, the ration $\mathrm{B}_{\mathrm{w}} / \mathrm{B}_{\mathrm{F}}$ is determined solely by the field line curvature on the open field lines in the midplane and thus it depends only on the separatrix and flux conserver shapes. This holds even when the open field lines are not straight in the midplane. It therefore seems reasonable to take the values of $B_{w} / B_{F}$ and $\langle\beta>$ determined in the $2 D$ equilibrium calculation, and plug them into the corrected diffuse profile model. For the trapped flux, the corrected diffuse profile is

$$
\phi_{t}^{*} \equiv \frac{1}{1-x_{s}^{2}}\left[\frac{x_{s}^{2}}{2}\right]\left[\frac{B_{f}}{B_{w}}\right]\left[1-\left(\frac{B_{w}}{B_{F}}<\beta>\right]^{2}\right]^{(1+\varepsilon) / 2} .
$$

The corrected fluxes (having a superscript ${ }^{*}$ ) in Table I.1 are reduced according to this formula. The corrected high flux limit $\phi_{\mathrm{H}}$ is obtained by taking $\varepsilon=0$. The fluxes $\phi_{\mathrm{t}}$ and $\phi_{\mathrm{l}}^{*}$ in Table I.1 are calculated assuming $\varepsilon=0.25$. The uncorrected fluxes are determined using the average beta condition for given $x_{s}$ and $B_{w}$. All of these fluxes are normalized with respect to the wall flux. The resulting confinement time obtained is $200 \mu \mathrm{sec}$. This is calculated using the approximate 
formula',

$$
\tau_{\phi}=60 \mu \sec 0.5(\phi(30.5)-\phi(90.5)) /[\phi(30.5)+\phi(90.5)] .
$$

which is a central finite difference approximation of the definition of $\tau_{\phi}$. Subsequent analysis of radial chord interferometry predicts $200 \pm 50 \mu \mathrm{sec}$ [5]. In this analysis model pressure profiles are fit to interferometer data. The interferometers respond to $\int \mathrm{nd} l$ where $\mathrm{n}$ is the particle density and $l$ is the distance along the interferometer beam. The fitted profiles are then used together with the assumption that $\mathrm{p}+\mathrm{B}_{z}^{2} /(8 \pi)$ is constant to infer the trapped flux. If profiles having the same form are fit to the data one arrives at the $200 \mu \mathrm{sec}$ figure. Between different model choices there is a large variation in the inferred trapped flux.

The increased estimate for $\tau_{\phi}$ reduced the needed field null anomaly factor from 8 to 4 . Possible effects due to time dependent profiles have been neglected in both the interferometric, and diamagnetic fits. In the next section it is shown that further reductions are possible when various profiles are considered.

\section{I.2 Profile effects}

It has been known for some time that profile effects, meaning the specific choice of $\mathrm{p}(\psi)$ in the MHD equilibrium model, could influence the deduction of the trapped flux. Previous to this work, no effort had been made to quantitatively assess the importance of such effects. In addition, it does not appear to have been widely realized that such effects could strongly influence the deduction of the field null resistivity without strongly influencing $\tau_{\phi}$.

Interest in the question as to whether the "high-flux" limit was valid began in the summer of 1986, when Spencer noted informally that there was a problem constructing the two dimensional analog of the high-flux FRC. He predicted that when one tried to approach the high flux limit in two dimensions the forces due to field line curvature near the field null would become important. He analysed a highly idealized model of an FRC having no toroidal effects and concluded that 
there really was no upper limit to the flux confined in an FRC with an observed value of $x_{s}$. At that time an early version of the MHD code was modified by changing the specification of the function $\mathrm{p}(\psi)$ so thc. a spike in the toroidal current density at the field null could be obtained. A 2-D MHD equilibrium having a trapped flux in excess of the high-flux limit was then computed. Subsequently, Spencer has made a more detailed study of the high-flux limit in two dimensions[6]. Results of that study are that the $\left(x_{s}, \beta_{s}\right)$ accessibility curve are now understood, and there may indeed be an upper limit to the trapped flux. This limit would be well above the earlier high flux limit and is due to a combination of the constraint of radial pressure balance and toroidal effects.

The work described in this report pursued the high flux equilibrium from a somewhat different point of view. As pointed out in the earlier report, the inference of the field null resistivity depends crucially on the value assumed from the field null current density. These high flux equilibria have very large currents at the field null, and consequently they will yield lower inferred field null resistivities. Manheimer and Finn[7] showed that in general it is possible to pose a transport problem that would cause peaking of the current at the field null. In fact, they present a similarity solution for an FRC in which trapped flux is dissipated and the field null resistivity is identically zero. That similarity solution had a singularity in the current at the field null. It was generally regarded as being a mathematical curiosity. Because of the similarity between the high flux solutions and their solution, it would seem that one can not rule out the high flux type of equilibrium a priori on physical grounds. The question is thus one of whether one can detect these equilibria with the diagnostic techniques that exist on the experiment.

In the remainder of this section it is shown that one can find equilibria having substantially different internal current density distributions but which are not distinguishable using the existing diagnostics (radial chord interferometry and external field measurements). This is a 
significant bit of information for people studying the theoretleal uspects of $F R C$ confinement. It means that when one looks at the fleld null for an anomalous Inter-spectes drag (l,e., an anomalous resistivity) or for bizarre kinetic effects (e.g., electron viscosity enhanced by stochastic particle orbits) one may be looking in the wrong place! There is presently no experimental evidence of a lack of understanding of the field null resistivity, Instend, it is the observed rate of decrease of the separatrix radius that is not understood.

Figure 1.3-I.5 present line plots of normalized pressure, magnetic field, and toroidal current density as a function of radius in the axial midplane for four different equilibria. These equilibria are denoted by a solid line, a dashed line, a dotted line and a dot-dashed line in the plots. One can see that these equilibria are similar in many respects, they have about the same separatrix radius and extemal magnetic field. They have very different toroidal current profiles. All of the profiles have a spike at the separatrix $\left(r_{s}\right.$ is about $16 \mathrm{~cm}$ ). Most of the current profiles have two spikes. The second spike occurs at the field null. As can clearly be seen from Figure 1.5, the current density at the field null can easily vary by a factor of 3 . It is likely that it can vary by more than this; however, no attempt to compute equilibria having larger currents at the field null was made. The external magnetic field probes are not capable of distinguishing between these equilibria because they are only sensitive to the separatrix shape. One hope for identifying these equilibria lay with the radial chord interferometers, which are used to provide profile information. Figure 1.6 shows the predicted interferometer signal as a function of impact parameter for the four equilibria. Also shown on this plot are data taken from 10mTorr LSM cperation when the passive mirrors were present. The discrepancy at zero impact parameter is to be expected because the equilibrium calculations were made for FRC's not under the influence of passive mirrors. The reason for the difference is that when the FRC leans on the mirrors, $<\beta>$ and $\beta_{s}$, the value of $\beta$ at the separatrix, are higher than would be the case if the 


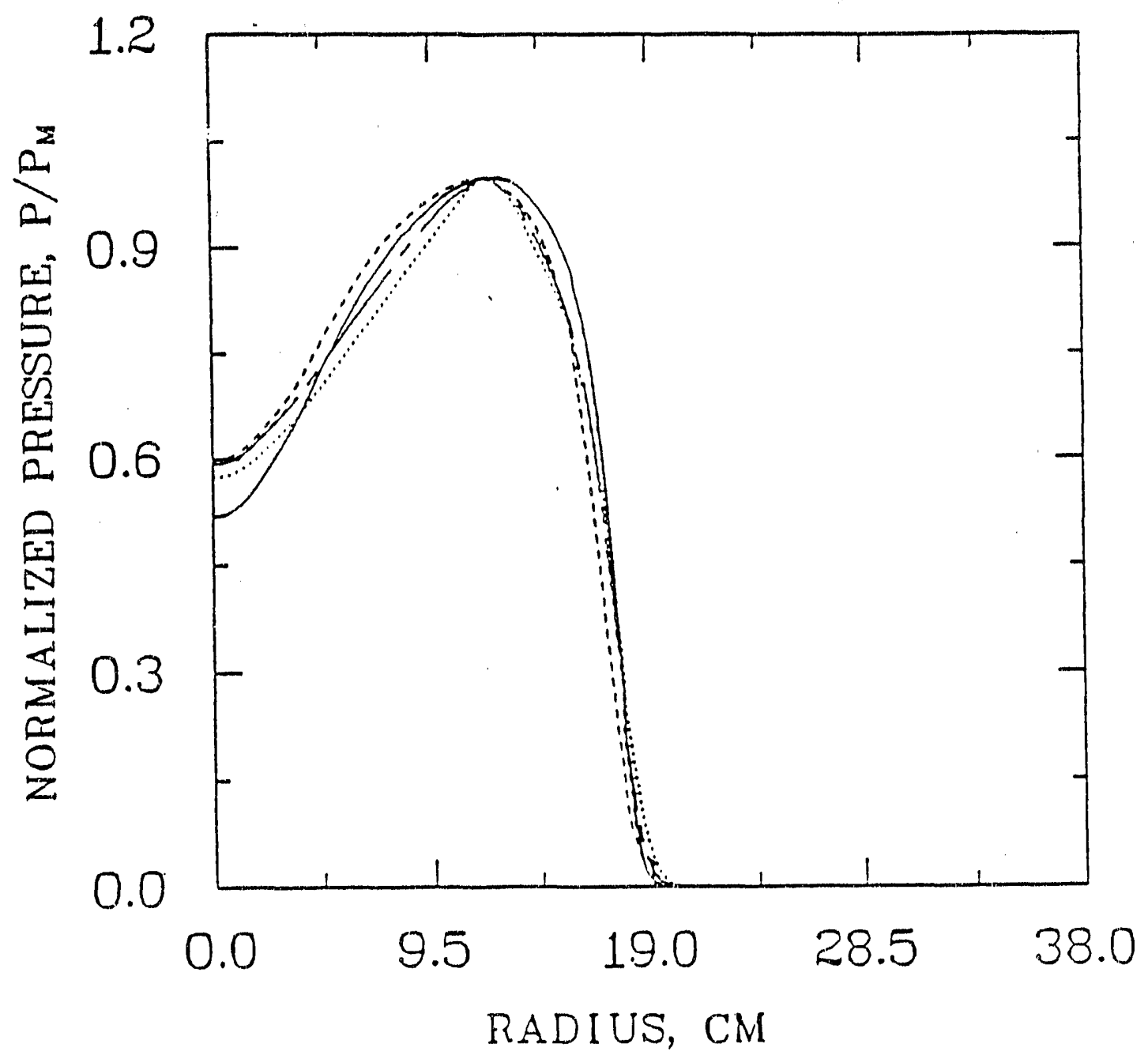

Figure I.3: Midplane pressure profiles for four different equilibria. 


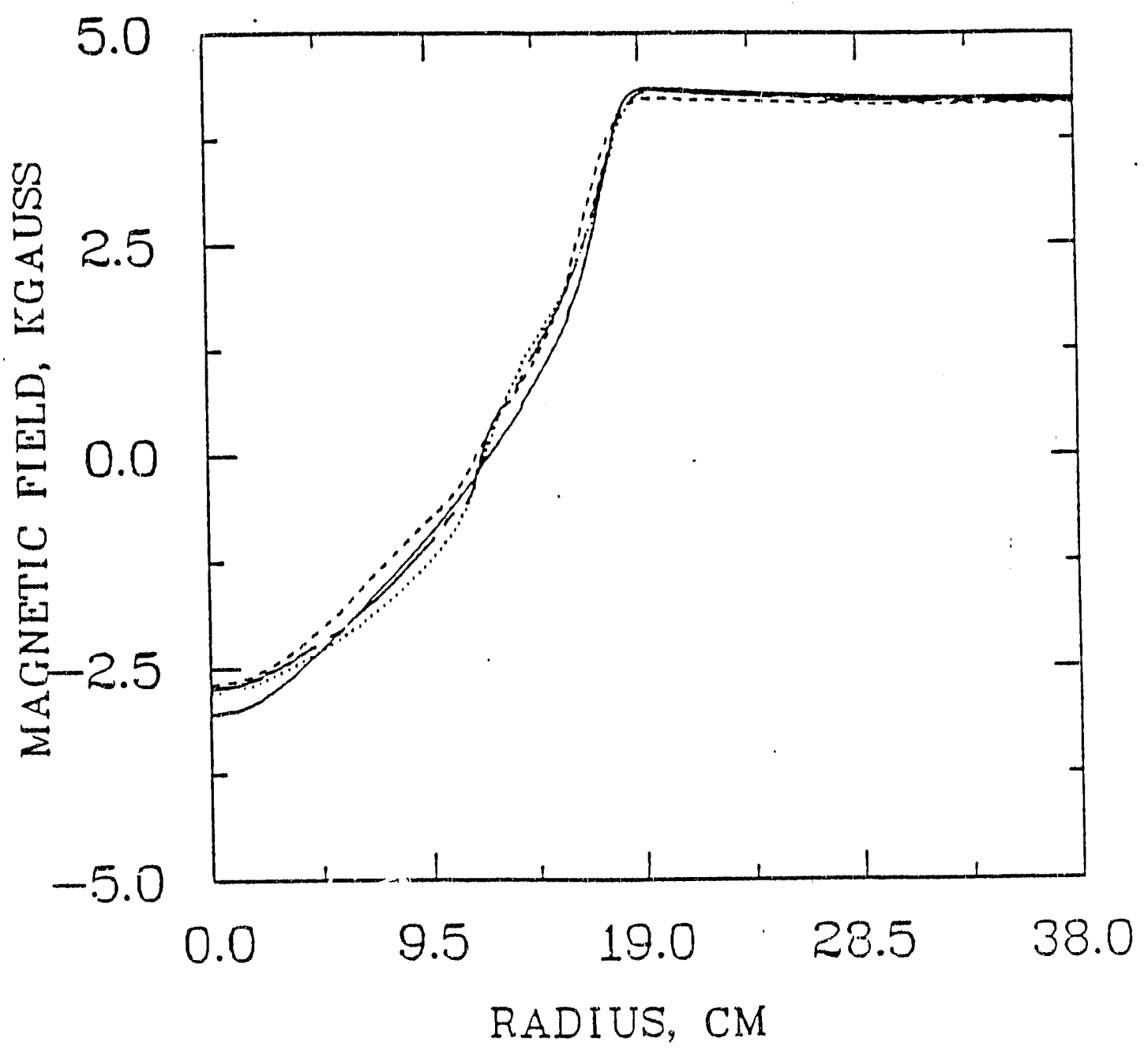

Figure 1.4: Midplane magnetic field profiels for four different equilibria. 


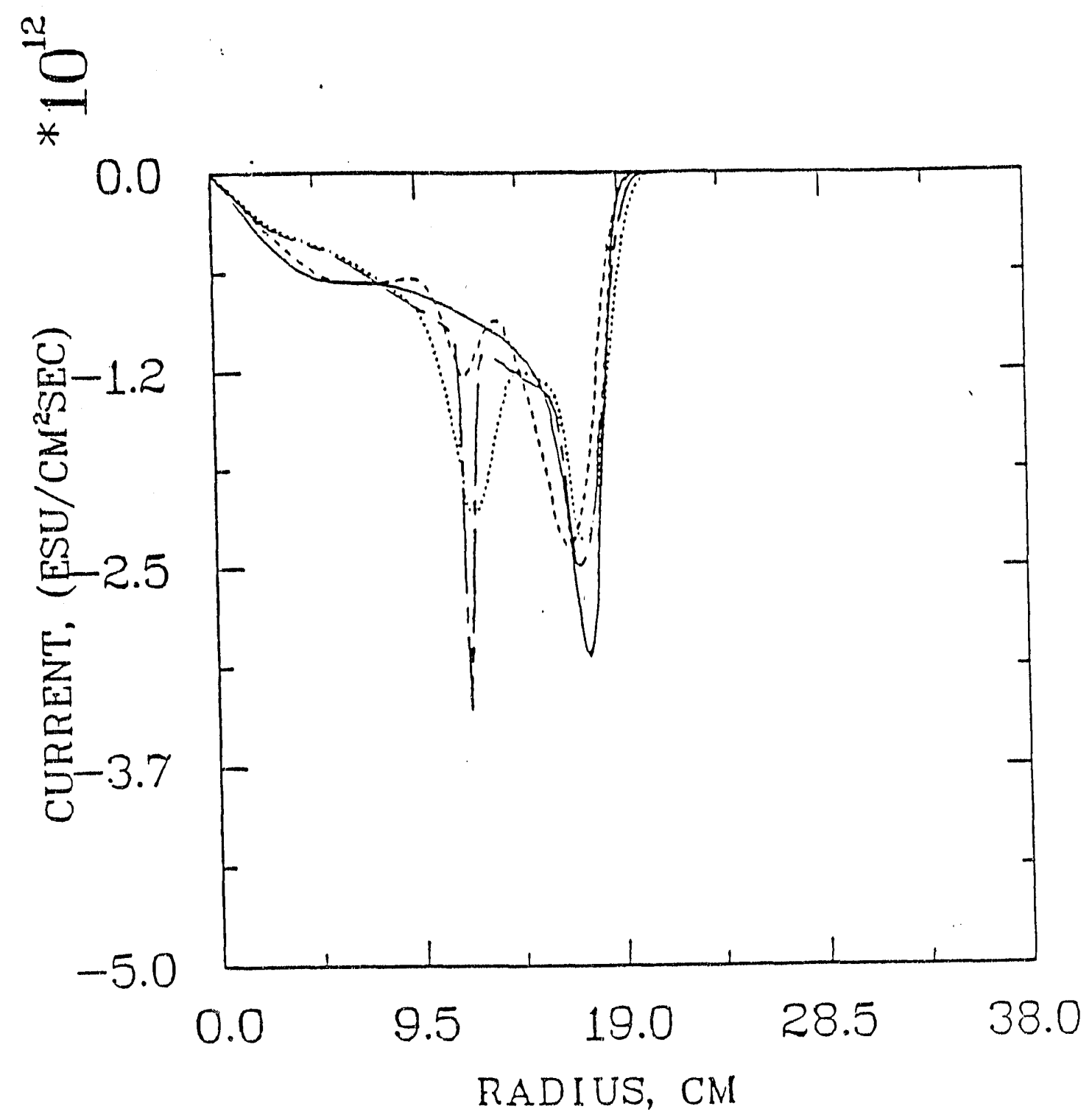

Figure I.5: Midplane toroidal current density profiles for four different equilibria. 


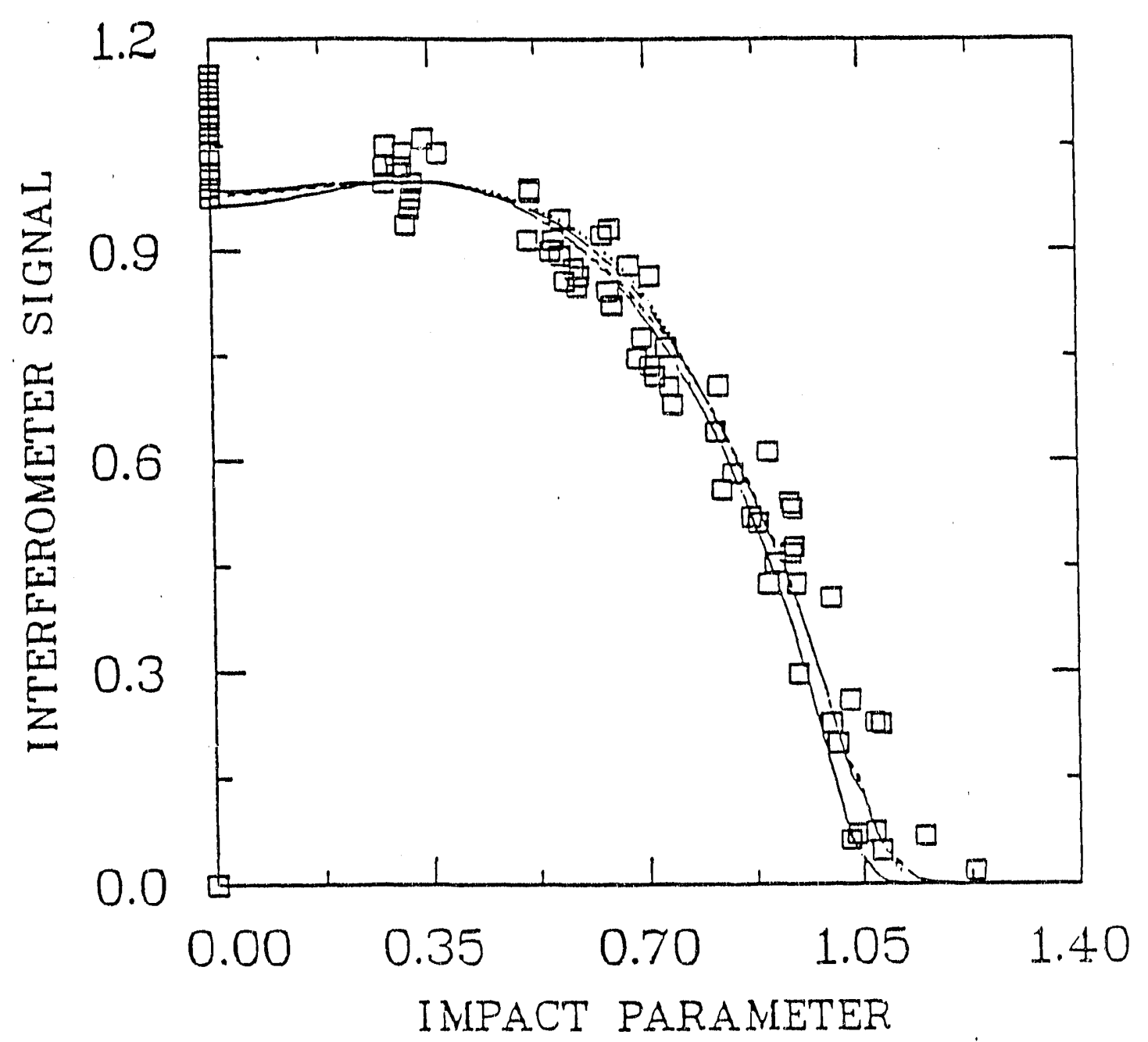

Figure I.6: Comparison of experimentally obtained interferometer data with predictions based on the four different equilibria. 
Table 1.2: Summary of the 4 equilibria used in the high-flux study. The curves in the previous comparison plots that correspond to the cases presented here are: 1) solid line, 2) dashed line, 3) dotted line and 4) dot-dashed line.

\begin{tabular}{ccccccc}
$\#$ & $\mathrm{x}_{\mathrm{s}}$ & $\phi_{\mathrm{o}} / \phi_{\mathrm{w}}$ & $\mathrm{z}_{\mathrm{s}}$ & $\beta_{s}$ & $\tau_{\text {tilt }}$ & $\varepsilon$ \\
\hline 1 & 0.453 & 0.039 & 1.79 & 0.52 & $6 \mu \mathrm{sec}$ & 0.07 \\
2 & 0.430 & 0.032 & 1.96 & 0.60 & $7 \mu \mathrm{sec}$ & 0.06 \\
3 & 0.444 & 0.044 & 2.30 & 0.57 & $6 \mu \mathrm{sec}$ & -0.09 \\
4 & 0.441 & 0.040 & 2.21 & 0.59 & $7 \mu \mathrm{sec}$ & -0.04
\end{tabular}

mirrors were not present. The zero impact parameter chord is sensitive to the particle density on the axis. Recall that the axis is also part of the separatrix; thus, modifying the pressure at the separatrix will usually modify the zero impact parameter chord (temperature is observed to be fai. $y$ constant over the plasma interior). For this reason, exact agreement between the computed curves and the experimental data is not to be expected. What is expected is that the scatter of the experimental data will be comparable with or without the murors. Clearly the scatter is too large to be able to experimentally determine which of the equilibria occurs in the experiment.

Therefore, it is safe to say that current .......ty at the field null is not known. Therefore, the inference of a field null resistivity is unwarranted.

So far the discussion has centered on the uncertainties in determining the field null current. There are also questions raised concerning the inference of the trapped flux, and hence the flux confinement time. Table I.2 summarizes some basic quantities of these equilibria. For the most part the numbers presented in the table are self explanatory. The quantity $\tau_{\text {ilt }}$ is presented here for compactness but is not discussed until the next section. The other new quantity is $z_{s}$, the ratio of the separatrix length to the wall radius. No effort was made to keep the lengths the same because for FRC's with these elongations $(\approx 4)$ the midplane profiles are not sensitive to the 
particular value of the length. The values for $\varepsilon$ are determined from the relation

$$
\phi_{o} / \phi_{w}=\frac{1}{1-x_{s}^{2}}\left[\frac{x_{s}}{\sqrt{2}}\right]^{3+\varepsilon},
$$

which is the dimensionless analog of the usual diffuse profile model. Clearly, $\varepsilon$ is not 0.25 . In fact, in two cases $\varepsilon$ is less than zero. This would not be possible if the high flux limit were truly a bound on the trapped flux.

One final point that should be mentioned, but cannot be addressed in an equilibrium study, is the possibility that the profiles change during the discharge. In the present reduction of experimental data, $\varepsilon$ is held constant. If the profiles are changing this is not appropriate. As an example of the potential important of this effect, support that the information code is correct in its indication that early in the discharge $\varepsilon=0.25$. Now suppose that due to some unknown transport process the profile evolves so that at the end of the discharge $\varepsilon \simeq 0$. For the typical in situ discharge, one can take the initial $x_{s}$ to be 0.45 at $20 \mu \mathrm{sec}$ and the final $x_{s}$ to be 0.40 at $60 \mu \mathrm{sec}$. Application of the usual diffuse profile model with $\varepsilon=0.25$ gives the trapped flux at the $20 \mu \mathrm{sec}, \phi_{\text {initial }}=0.03 \phi_{\text {wall }}$ and the trapped flux at the $60 \mu \sec \phi_{\text {final }}=0.02 \phi_{\text {wall }}$. Using these two points to approximate the logarithmic derivative of $\phi$ with respect to time one obtains approximately a $100 \mu \mathrm{sec}$ confinement time. If one assumes $\varepsilon=0$ at the final time the result is $\phi_{\text {linal }}=0.027 \phi_{\text {wall }}$ with a resulting confinement time of $370 \mu$ sec. $^{3}$ Here again there is a large uncertainty in the flux confinement time, and hence in the inferred field null resistivity. Here it is tacitly assumed that the wall flux is constant over the discharge. For LSM the wall flux decay time is about $400 \mu \mathrm{sec}$. This is not very much longer than the trapped flux decay times examined; it should be included to get an accurate value of the decay time. Since the point of

3. Note that for all 4 equilibria used in the high flux study $\varepsilon$ is closer to zero than to 0.25 . A current spike at the field null is not even necessary for this effect to be large. 
this discussion is that the usual formulae must be modified, it suffices to assume an infinite wall flux decay time. The wall flux decay affects only the absolute values of the decay rate and not the fact that changing profiles can lead to a change in the inferred flux.

To summarize this section, equilibria have been found that are not distinguishable by present experimental diagnostics. These equilibria imply very different values for the field null resistivity. It is not known that a problem exists near the field null. More work with fluid transport is needed to assess the possibility that the observed decrease in $x_{s}$ is due to enhanced resistivity in portions of the FRC other than the field null. Note that this invokes no new assumptions; it is generally recognized that the transport processes are unknown.

\section{I.3 Rotation effects}

During the course of the current work the effects of bulk plasma rotation were also examined. As a result of work with the 1-D kinetic equilibrium problem it was clear that one could develop a rotating model for 2-D FRC equilibria that was significantly more general than those that were in use. Specifically, one could use a fluid model for the electrons that would allow the same level of freedom in the choice of $\mathrm{p}(\psi)$ that is enjoyed with the MHD model. Previous models of rotating FRC's (primarily those of Shumaker) suffered either from the assumption that the electrons rotated rigidly or that the ion kinetic distribution function was linear in $\mathrm{p}_{\theta}$. In the former case, only a very limited range of pressure profiles is obtainable. In the latter case, the distribution function is only reasonable for low rotation rates. The kinetic model presented in the earlier report[1] suffered from neither of these shortcomings, but was expected to be very expensive to solve. By restricting the ions to have the rigid rotor profile and taking the electrons, as fluid one arrives at an affordable model. This rigid rotor model is described in Ref.[1]. 
Examples of the effect that rotation has on the basic FRC equilibrium are also presented in

Reference[1]. In addition to the equilibrium effects (which turn out to be ignorable for present experiments), stability has been studied. Rotating equilibria were computed and used as input to a 3-D MHD initial value code ${ }^{4}$ in a collaborative effort with Richard Milroy of STI, and Robert Bishop and Daniel Barnes of SAIC-Austin. The code solves the resistive MHD equations in three dimensions. No effect was found when the FRC spins slowly, when the Mach number $M \equiv(1) r_{s} / v_{t}<<1$ where $\omega$ is the angular frequency of the rigid rotation and $v_{t}$ is the thermal speed. When the FRC rotates quickly enough, $M=1$, the tilt changes character from being an internal mode to being an external mode. [8] This meant that it might be possible to stabilize the mode by placing the FRC in strong mirrors. Subsequently the ROT206 equilibrium was examined. This equilibrium code was also presented in Ref.[1] as an example of the use of the rigid rotor equilibrium code. This equilibrium did not disrupt. Figure I.7 shows pressure contours at the end of the simulation for the ROT206 equilibrium. Tnis is encouraging because the prediction that a large, MFID-like FRC should tilt is perceived to be one of the major obstacles to overcome in scaling the FCR device to the fusion reactor regime. The rotation rate used corresponds to $M=1.14$ which is very large. Continued work is needed to see how this result depends on pressure profiles, mirrors and/or length.

\section{I.4 Stability}

Some mention of stability is made in the preceding summary of rotational effects. In this section the results of an examination of resistive tilt stability are presented. For this study only the pressure profile is changed. The equilibria considered are those examined in the section dealing with profile effects. The study of tilt stability of the FRC is not new; however, prior to the present study only a limited ability to generate FRC equilibria existed. Primarily the

4. FRCD, version $\mathrm{f} 3 \mathrm{~d} 25.8$, proprictary software of SAIC 
limitation was in the choice of the function $\mathrm{p}(\psi)$. The Hewett-Spencer code could not reliably find equilibria for situations in which $d p / d \psi$ was sharply peaked at the separatrix. Shumaker's code generally found equilibria with very sharply peaked $d p / d \psi$. This was a result of logarithmic singularity in the entropy formulation near the separatrix. Rather than analytically removing the singularity, he avoided dealing with the singularity by choosing the quadrature points in his finite element solution method such that the separatrix was never sampled. As a result, the toroidal current forms a vry narrow very spike at the separatrix. 'Thus a clean study' of profile effects was not possible.

In addition, much of the previous MHD stability analysis was done before any of these codes existed. Only a very limited class of approximate analytic equilibria was available for stability analysis. Therefore the original survey of FRC stability should be expanded to include the greater variety of equilibria now attainable.

In pursuit of the question as to whether the high fux type equilibria can be reasonably expected to exist in the experiment, the resistive MHD tilt stability for these equilibria was examined. No striking difference in the growth times of the tilt modes for these different equilibria is observed, and thus conclude that there is no reason based on stability to rule out the high flux type of equilibrium. There is a difference in the basic structure of the modes.

The tilt stability results are summarized in Table I.2 and Figures I.7-I.9. The striking feature is that the structure of the tilt is very different in the two cases (see Figures I.7-I.8) although the kinetic energy of the tilt (the $n=1$ modal kinetic energy, where $n$ is the toroidal mode number) grows at about the same rate, as seen from Figure I.9. These runs were made with an initial $n=$ 1 perturbation on the velocity field of about 1 percent of the thermal velocity. Other runs with a 0.01 percent perturbation exhibited a well defined linear growth phase. The growth times $\tau_{\text {till }}$ presented in Table I.2 were determined from those runs. In all of these cases, the growth 

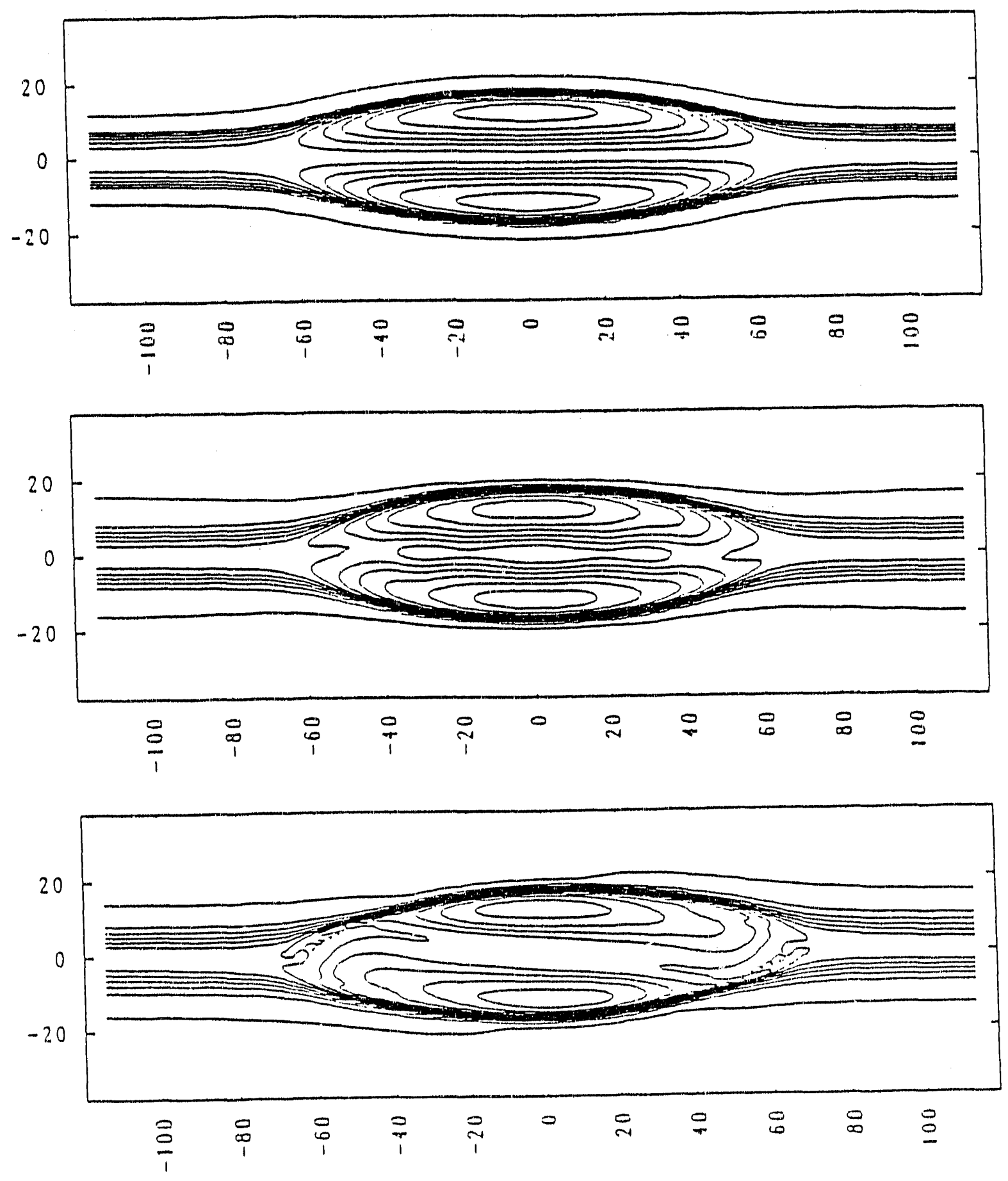

Figure 1.7: Pressure contours for case 1 of the high flux study as three different times, a) $0 \mu \mathrm{sec}$, b) $10 \mu \mathrm{sec}$ and c) $20 \mu \mathrm{sec}$. 

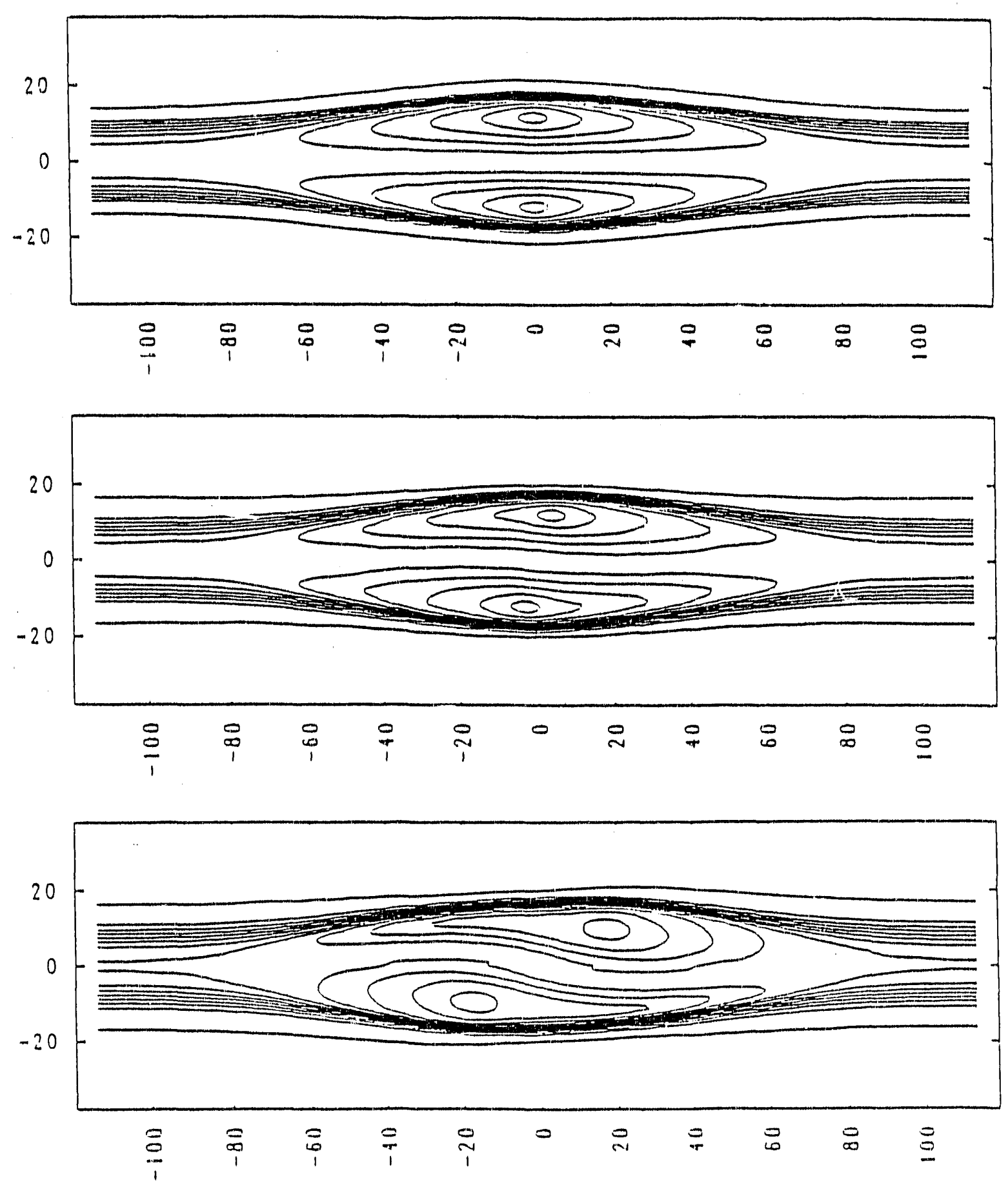

Figure I.8: Pressure contours for case 3 of the high flux study at three different times, a) $0 \mu \mathrm{sec}$, b) $1.0 \mu \mathrm{sec}$ and c) $20 \mu \mathrm{sec}$. 

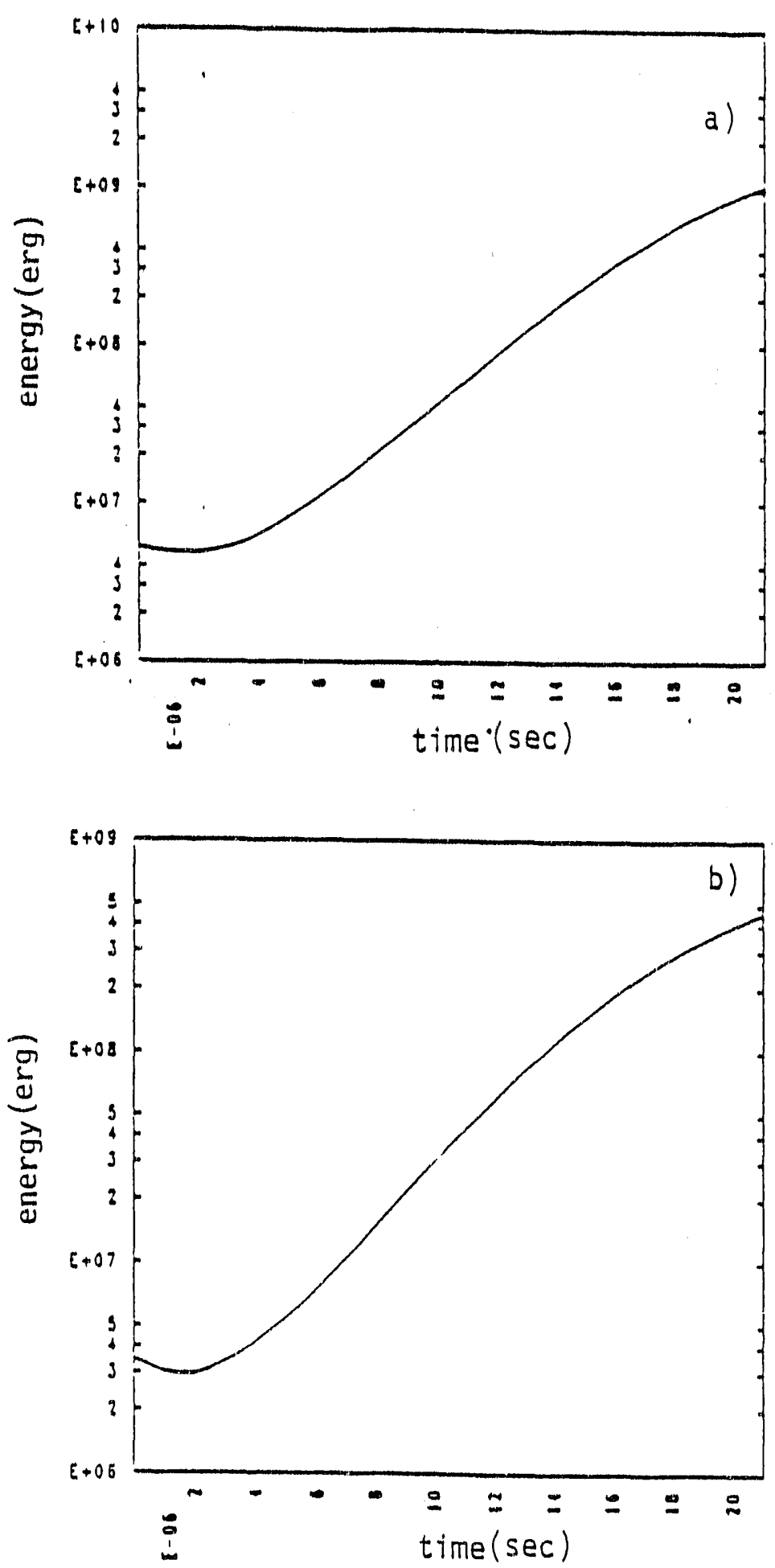

Figure I.9: Kinetic energy of the $m=1$ mode vs time for: a) case 1, and b) case 3 of the high flux study. 
times are about the same. FRC's are not presently observed to tilt. Debate and study continues as to'what the stabilizing mechanism not found in ideal MHD theory is. Two possibilities that have been proposed are kinetic stabilization and stabilization by micro-turbulence. The change in structure does suggest that if the tilt is indeed stabilized by some kinetic or micro-turbulent effect, or even if the mode saturates in some non-linear fashion, such stabilization may be pressure profile sensitive.

\section{THE 1-D LINEAR STABILITY}

The goal of the stability calculations at this time is to determine the eigenvalues and eigenfunctions of the linearized Vlasov-Maxwell system of equations. Briefly, the perturbed distribution function is expanded in terms of the functions, $w_{r}$, defined by the following eigenvalue problem:

$$
\left[\mathrm{w}_{\mathrm{r}}, \mathrm{H}_{\mathrm{O}}\right]=\mathrm{i} \mu_{\mathrm{r}} \mathrm{w}_{\mathrm{r}}
$$

where $\mathrm{H}_{0}=$ single-particle Hamiltonian in the equilibrium fields, and

$$
[A, B]=\sum_{i=1}^{3}\left\{\frac{\partial A}{\partial q_{i}} \frac{\partial B}{\partial p_{i}}-\frac{\partial A}{\partial p_{i}} \frac{\partial B}{\partial q_{i}}\right\},
$$

where $\left\{q_{i}\right\}_{i=1}^{3}$ are the position coordinates in the Hamiltonian and $\left\{p_{i}\right\}_{i=1}^{3}$ are the respective conjugate momenta. The $\mathrm{w}_{\mathrm{r}}$ are called the Liouville eigenfunctions. The expansion in $\mathrm{w}_{\mathrm{r}}$ leads to a system of integro-differential equations represented by

$$
\text { where } \hat{\Phi}_{1}=\left[\begin{array}{l}
\hat{\phi}_{1} \\
\vec{A}_{1}
\end{array}\right] \text { is an array a Laplace-transformed perturbation potentials, }
$$

and $\omega$ is the complex frequency. To solve Eqn. II.3, we employ a Spline-Galerkin method to form a discrete system of linear equation depending on the complex parameter $\omega$. Writing

$$
\phi_{n}(\vec{r}) \equiv \sum_{n=1}^{N} \alpha_{n} \eta_{n}(\vec{r})
$$

we are lead to the discrete system 


$$
\text { where } \vec{\alpha}=\left[\begin{array}{c}
\alpha_{1} \\
\cdot \\
\cdot \\
\alpha_{n}
\end{array}\right]=\text { array unknown coefficients. }
$$

The corresponding dispersion relation is clearly

$$
\operatorname{Det}\left|D_{\mathrm{nn}^{\prime}}(\omega)\right|=0
$$

The goal of the stability code under development, IDSTAB.f, is to solve Eqns. II.6 and II.5.

The next three sections describe the progress made towards that end in the past six months.

1DSTAB.f is a 1-D Vlasov stability code being developed at Purdue on a Gould NP-1. It is being written in Fortran 77 and is portable. Currently, it uses Purdue ECN libraries to do plotting and output, but can be easily converted to operate on any of the MFENET CRAY's.

II.1 Representing $\mathrm{D}_{\mathrm{nn} n^{\prime}}$ with less numerical computation

A discrete representation of the operator $D(\omega)$ is given by

$$
D_{n n^{\prime}}=\Lambda_{\mathrm{rn} n^{\prime}}(\omega)-\sum_{\mathrm{r}} \frac{K_{\mathrm{r}} j_{\mathrm{nr}}^{*} j_{n^{\prime} \mathrm{r}}}{\left[\mu_{\mathrm{r}}-\omega\right]}
$$

The pertinent definitions in Eqn. II 7 are

$$
\begin{aligned}
& \mu_{\mathrm{T}}=1 \Omega_{\mathrm{T}}\left(\mathrm{H}, \mathrm{p}_{\theta}, \mathrm{p}_{\mathrm{z}}\right)+\mathrm{m} \Omega_{\theta}\left(\mathrm{H}, \mathrm{p}_{\theta}, \mathrm{p}_{\mathrm{z}}\right)+\frac{\mathrm{k}}{\mathrm{m}} \mathrm{p}_{\mathrm{z}}, \\
& \Omega_{\mathrm{T}} \quad=\quad \text { radial bounce frequency, } \\
& \Omega_{\theta} \quad=\text { angular frequency of the motion, } \\
& \mathrm{m}=\text { azimuthal mode number, } \\
& \mathrm{k}=\text { axial mode number, } \\
& \Lambda_{\mathrm{nn} \mathbf{n}^{\prime}}=\text { the matrix that results when Maxwell's } \\
& \text { equations are discretized using the } \\
& \text { Spline-Galerkin method, }
\end{aligned}
$$

and $\sum_{\mathrm{r}}=\int \mathrm{dp}_{\theta} \int \mathrm{dH} \int \mathrm{dp} \sum_{\mathrm{z}} \sum_{i=-\infty}^{\infty} \sum_{\mathrm{a}}$ 
where $\sum_{a}$ represents an inherent summation of all possible orbits for a given $\left(H, \mathrm{P}_{\theta}, \mathrm{P}_{z}\right)$ and

$$
\mathrm{K}_{\mathrm{r}}=\mu_{\mathrm{r}} \frac{\partial \mathrm{f}_{\mathrm{o}}}{\partial \mathrm{H}_{\mathrm{o}}}=\mathrm{m} \frac{\partial \mathrm{f}_{\mathrm{o}}}{\partial \mathrm{p}_{\theta}}+k \frac{\partial \mathrm{f}_{\mathrm{o}}}{\partial \mathrm{p}_{\mathrm{z}}},
$$

where $f_{0}$ is the equilibrium distribution function. The $f_{0}$ has the form

$$
f_{0}=\beta\left(p_{\theta}\right) e^{-H / \alpha\left(p_{\theta}\right)},
$$

where $\alpha$ and $\beta$ are determined by the equilibrium code.

The first goal was to eliminate as much of the numerical computation as possible involved in the $\sum_{r}$. Most important is the infinite summation. The quantity $j_{n_{r}}^{*} j_{n^{\prime} r}$ has a $p_{z}$ dependence that is of a polynomial form. Hence, we hoped to do the $\mathrm{p}_{\mathrm{z}}$ integration analytically as well. Since $\Omega_{\mathrm{r}}$ and $\Omega_{\oplus}$ have a $p_{z}$ dependence but are computed numerically, we changed the variables of integration: $\left(\mathrm{p}_{\theta}, \mathrm{p}_{z}, \mathrm{H}\right) \rightarrow\left(\mathrm{p}_{\theta}, \mathrm{p}_{z}, \mathrm{H}_{\perp}\right)$.

The Jacobian of the transformation is unity, and the only resulting $p_{z}$ dependence is in $f_{0}$ :

$$
f_{0} \rightarrow f_{0}^{1}=\beta\left(p_{\theta}\right) \exp \left[-H \downarrow / \alpha\left(p_{z}\right)-\frac{p_{z}^{2}}{\partial m \alpha\left(p_{\theta}\right)}\right],
$$

thus, for the $k=0$ case, the $p_{z}$ integration is easily performed. For $k \neq 0$ we use the other form of $\mathrm{D}_{\mathrm{nn}}(\omega)$ given by

$$
D_{n n^{\prime}}(\omega)=\Lambda_{n n^{\prime}}(\omega)-\int_{-\infty}^{\infty} d \mu \frac{S_{n n^{\prime}}(\mu)}{(\mu-\omega)}
$$

where $S_{n n^{\prime}}(\mu)=\sum_{r} \delta\left(\mu-\mu_{r}\right) K_{r} j_{n r}^{*} j_{n^{\prime} r}$.

This form conveniently removes the $\omega$-dependence from the orbit integrations and allows the $\mathrm{p}_{z}$ integration to be performed via the $\delta$-function. Further, the resonant dominator in Eqn. II.7 is removed. The matrix $\mathrm{S}_{\mathrm{nn} n^{\prime}}$, called the Dynamic Spectral Matrix, is computed once for various values of $\mu$ and the integration to get $D_{n n^{\prime}}(\omega)$ can be done numerically using various 
interpolation formulas, provided the restrict $\operatorname{Im}(\omega) \neq 0$. Since we are interested in $\operatorname{Im}(\omega)>0$ this poses no restrictions.

Once the $\mathrm{p}_{z}$ integrations are done, the infinite summation results in trigonometric and delta functions for the $k=0$ case and transcendental functions called $\theta_{2}$ functions for $k \neq 0$. The $\theta_{2}$ functions can be easily computed to machine precision. The $\sum_{\mathrm{r}}$ has been reduced to two numerical integrations and a finite summation.

\section{II.2 Regularity conditions}

Since the Maxwell's equations are being applied to a cylindrical geometry, one must be concerned about the behavior of the computation near the origin. Lewis and Schwarzmeier (unpublished) approached this problem several years ago and developed two methods to attack this problem. The first was to determine the asymptotic form of the distribution function near $r=0$. The second was to find basic functions that guarantee that the computed potentials would be infinitely continuous near $r=0$.

The latter method is employed in IDSTAB.f. It was chosen because to within 4 unknown coefficients (which are determined in the code) the exact method and the latter method are correct to $6^{\text {th }}$ order.

\section{II.3 Boundary Conditions}

The gauge choice made in determining the form of $\Lambda_{\mathrm{rn} n^{\prime}}$ is the radial component of the perturbed magnetic potential, $A_{1 r}=0$. While this greatly simplifies the problem, by reducing the number of functions that must be found, it results in frequency dependent boundary conditions. This required the redefinition of the basic functions representing the potentials near the boundary. 
II.4 Work currently being done.

Currently we are testing the code for the case where no plasma is present. The result must be TE and TM wave guide modes. The goal of this computation is two-fold. It serves as a benchmark of the $\Lambda_{\mathrm{nn}}{ }^{\prime}(\omega)$ portion of the dispersion relation, and it also gives a useful test bed for developing a complex equation solver.

\section{RESEARCH STAFF}

This project involved two graduate students, Bob Webster and Pete Papanikolaou at Purdue University under the supervision of Professor Chan Choi in the School of Nuclear Engineering. Bob Webster has since completed his Ph.D. study on this 2-D equilibrium FRC and is currently a staff member at the Los Alamos National Laboratory. Pete is continuing on his work on the linear stability study in FRC. This research is closely collaborated with the on-going FRC project at Los Alamos National Laboratory, in particular, with Dr. Ralph Lewis. 


\section{REFERENCES}

[1] C. Choi, Non-Local Kinetic Transport Studies of an FRC, Annual Report to DOE, Purdue University, Report No. DOE-53250-002 (June 1988).

[2] R.L. Spencer and M. Tuszewski, Experimental and computational equilibria of fieldreversed configurations, Phys. Fluids, 28:1810, 1985.

[3] M. Tuszewski and R.L. Spencer, Equilibrium properties of short field-reversed configurations. Phys. Fluids, 29 (11):3711, 1986.

[4] R.L. Spencer, M. Tuszewski and R.K. Linford, Adiabatic compression of elongated field-reversed configurations. Phys. Fluids, 26(6):1564, 1983.

[5] R.E. Chrien and S. Okada, Field-reversed configuration profiles and resistivities inferred from the radial line-integral density profile. Phys. Fluids, 30:3574, 1987.

[6] R.L. Spencer, private communication.

[7] W.M. Manheimer and J.M. Finn; Transport studies in reversed field theta pinches. NRL Memorandum report 4348, 1980.

[8] R.D. Milroy, D.C. Barnes, R.C. Bishop, D.D. Schnack, and R.B. Webster, MHD studies of the tilt mode in a field-reversed configuration. Bulletin of the American Physical Society, 1987. 

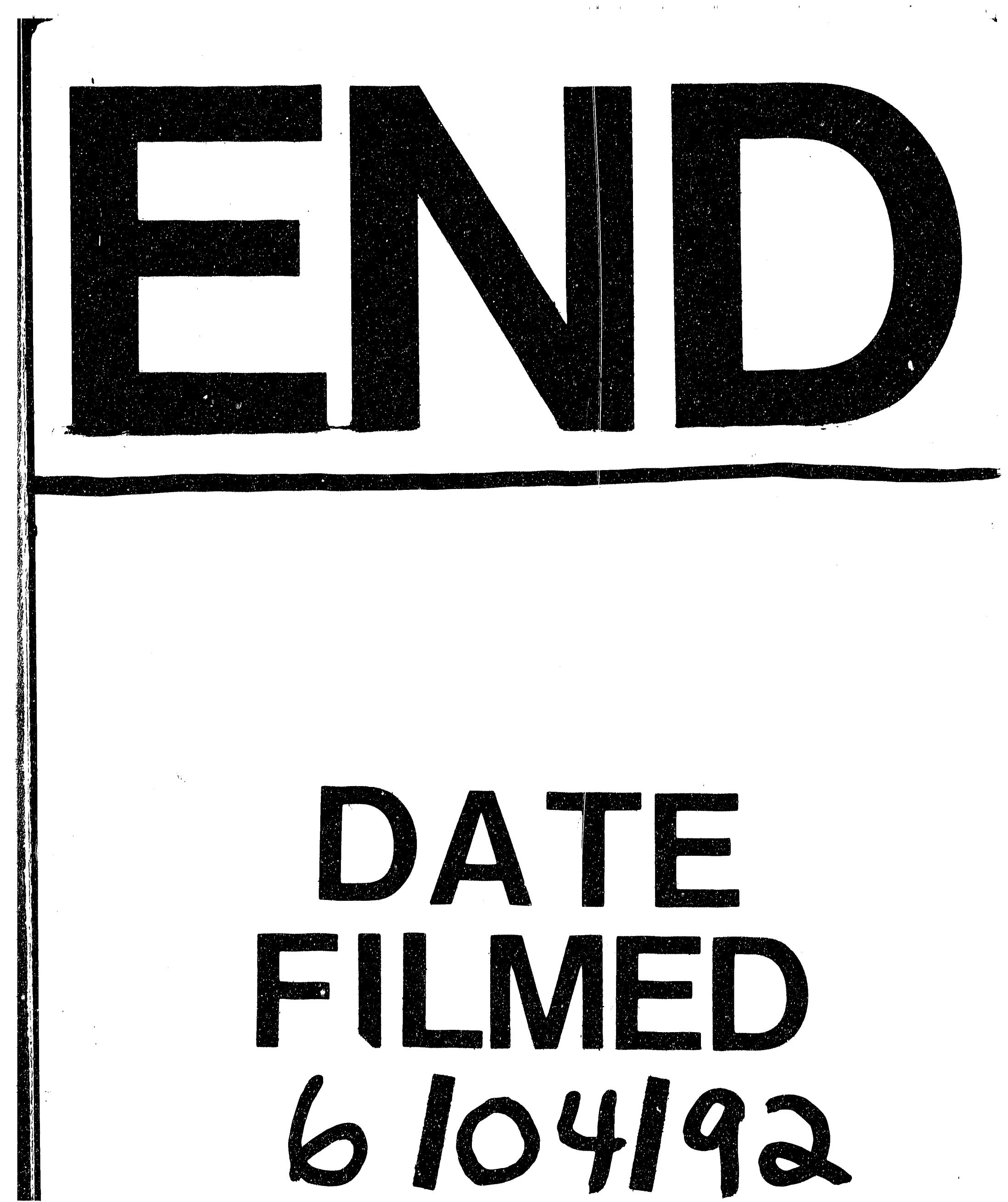
